University of Nebraska - Lincoln

DigitalCommons@University of Nebraska - Lincoln

Robert Streubel Papers

Research Papers in Physics and Astronomy

$5-1-2018$

\title{
Hartmann characterization of the PEEM-3 aberration-corrected X- ray photoemission electron microscope
}

\author{
A. Scholl \\ Lawrence Berkeley National Laboratory, a_scholl@lbl.gov
}

M. A. Marcus

Lawrence Berkeley National Laboratory

A. Doran

Lawrence Berkeley National Laboratory

J. R. Nasiatka

Lawrence Berkeley National Laboratory

A. T. Young

Lawrence Berkeley National Laboratory

See next page for additional authors

Follow this and additional works at: https://digitalcommons.unl.edu/physicsstreubel

Part of the Atomic, Molecular and Optical Physics Commons, Condensed Matter Physics Commons, and the Other Physics Commons

Scholl, A.; Marcus, M. A.; Doran, A.; Nasiatka, J. R.; Young, A. T.; MacDowell, A. A.; Streubel, R.; Kent, N.; Feng, J.; Wan, W.; and Padmore, H. A., "Hartmann characterization of the PEEM-3 aberration-corrected Xray photoemission electron microscope" (2018). Robert Streubel Papers. 12.

https://digitalcommons.unl.edu/physicsstreubel/12

This Article is brought to you for free and open access by the Research Papers in Physics and Astronomy at DigitalCommons@University of Nebraska - Lincoln. It has been accepted for inclusion in Robert Streubel Papers by an authorized administrator of DigitalCommons@University of Nebraska - Lincoln. 


\section{Authors}

A. Scholl, M. A. Marcus, A. Doran, J. R. Nasiatka, A. T. Young, A. A. MacDowell, R. Streubel, N. Kent, J. Feng, W. Wan, and H. A. Padmore 


\title{
Hartmann characterization of the PEEM-3 aberration-corrected X-ray photoemission electron microscope
}

\author{
A. Scholl a,*, M.A. Marcus ${ }^{\mathrm{a}}$, A. Doran ${ }^{\mathrm{a}}$, J.R. Nasiatka ${ }^{\mathrm{a}}$, A.T. Young ${ }^{\mathrm{a}}$, A.A. MacDowell ${ }^{\mathrm{a}}$, \\ R. Streubel ${ }^{b}$, N. Kent ${ }^{\mathrm{b}, c}$, J. Feng ${ }^{\mathrm{a}}$, W. Wan ${ }^{\mathrm{a}}$, H.A. Padmore ${ }^{\mathrm{a}}$
}

a Advanced Light Source, Lawrence Berkeley National Laboratory, 1 Cyclotron Road, Berkeley CA 94720, USA

${ }^{\mathrm{b}}$ Materials Sciences Division, Lawrence Berkeley National Laboratory, 1 Cyclotron Road, Berkeley CA 94720, USA

${ }^{\mathrm{c}}$ Department of Physics, University of California, Santa Cruz, Santa Cruz, CA 95064, USA

\section{A R T I C L E I N F O}

Article history:

Received 6 December 2017

Revised 6 March 2018

Accepted 9 March 2018

Available online 10 March 2018

\begin{abstract}
A B S T R A C T
Aberration correction by an electron mirror dramatically improves the spatial resolution and transmission of photoemission electron microscopes. We will review the performance of the recently installed aberration corrector of the X-ray Photoemission Electron Microscope PEEM-3 and show a large improvement in the efficiency of the electron optics. Hartmann testing is introduced as a quantitative method to measure the geometrical aberrations of a cathode lens electron microscope. We find that aberration correction leads to an order of magnitude reduction of the spherical aberrations, suggesting that a spatial resolution of below $100 \mathrm{~nm}$ is possible at $100 \%$ transmission of the optics when using $\mathrm{x}$-rays. We demonstrate this improved performance by imaging test patterns employing element and magnetic contrast.
\end{abstract}

Published by Elsevier B.V.

\section{Introduction}

X-ray photoemission electron microscopy (X-PEEM) is a synchrotron based technique that allows researchers to image the morphology and the magnetic and chemical properties of surfaces, artificially grown thin films and minerals with nanometer-scale spatial resolution. X-PEEM has led to striking scientific discoveries in the area of materials sciences and physics, e.g., the observation of nanoscale domains in antiferromagnetic oxides [1] and the detection of the picosecond dynamics of magnetic vortex cores [2]. $\mathrm{X}$-PEEM is an $\mathrm{X}$-ray spectromicroscopy technique, combining spectroscopic sensitivity via the near edge $\mathrm{x}$-ray absorption fine structure (NEXAFS) effect with lateral resolution provided by electron microscopy $[3,4]$. In an X-PEEM instrument monochromatic X-rays from a synchrotron illuminate the sample and photoelectrons and secondary electrons are collected and imaged by the electron optics. The sample acts as a cathode, and the emitted electrons are accelerated rapidly towards the objective lens by an electrical field of $15-20 \mathrm{kV}$ over about $2 \mathrm{~mm}$. The spatial resolution of X-PEEM microscopes is usually reported to be near $20 \mathrm{~nm}[5,6]$, but is in practice often in the $50-100 \mathrm{~nm}$ range for objects with weak contrast. Compared to other cathode lens techniques the relatively poor resolution is a consequence of the wide energy and transverse mo- mentum distributions of the emitted electrons, resulting in blur caused by the chromatic and spherical aberrations of the microscope lenses. To reach high resolution, small contrast apertures need to be inserted that reduce the contribution of off-axis electrons but also drastically lower the transmission of the microscope. This leads to long exposure times and potentially higher radiation damage of sensitive samples. Increasing the photon flux to compensate for the inefficient microscope optics can lead to increased space charge blurring of images, further reducing the resolution.

Various approaches have been tried to reduce the impact of spherical and chromatic aberrations, for example the use of a dynamic correcting lens [7], coating the sample with diamondoid nanoclusters acting as an energy filter [8], or, as discussed here, the correction of the lowest order chromatic and spherical aberrations of the objective lens and accelerating field by an electron mirror [9-11]. The latter method has found broader use and has led to a spectacular improvement of the ultimate resolution of low energy electron microscopes (LEEMs) and UV-PEEMs using ultraviolet excitation. For X-PEEM's the progress has been slower but first experiments showed improved resolution but also a strong sensitivity to space charge effects [12]. We will discuss commissioning results of the aberration corrector of the PEEM-3 microscope, which is located at beamline 11.0.1 at the Advanced Light Source (ALS), a soft $\mathrm{x}$-ray undulator beamline with variable polarization.

\footnotetext{
* Corresponding author.

E-mail address: a_scholl@lbl.gov (A. Scholl).
} 


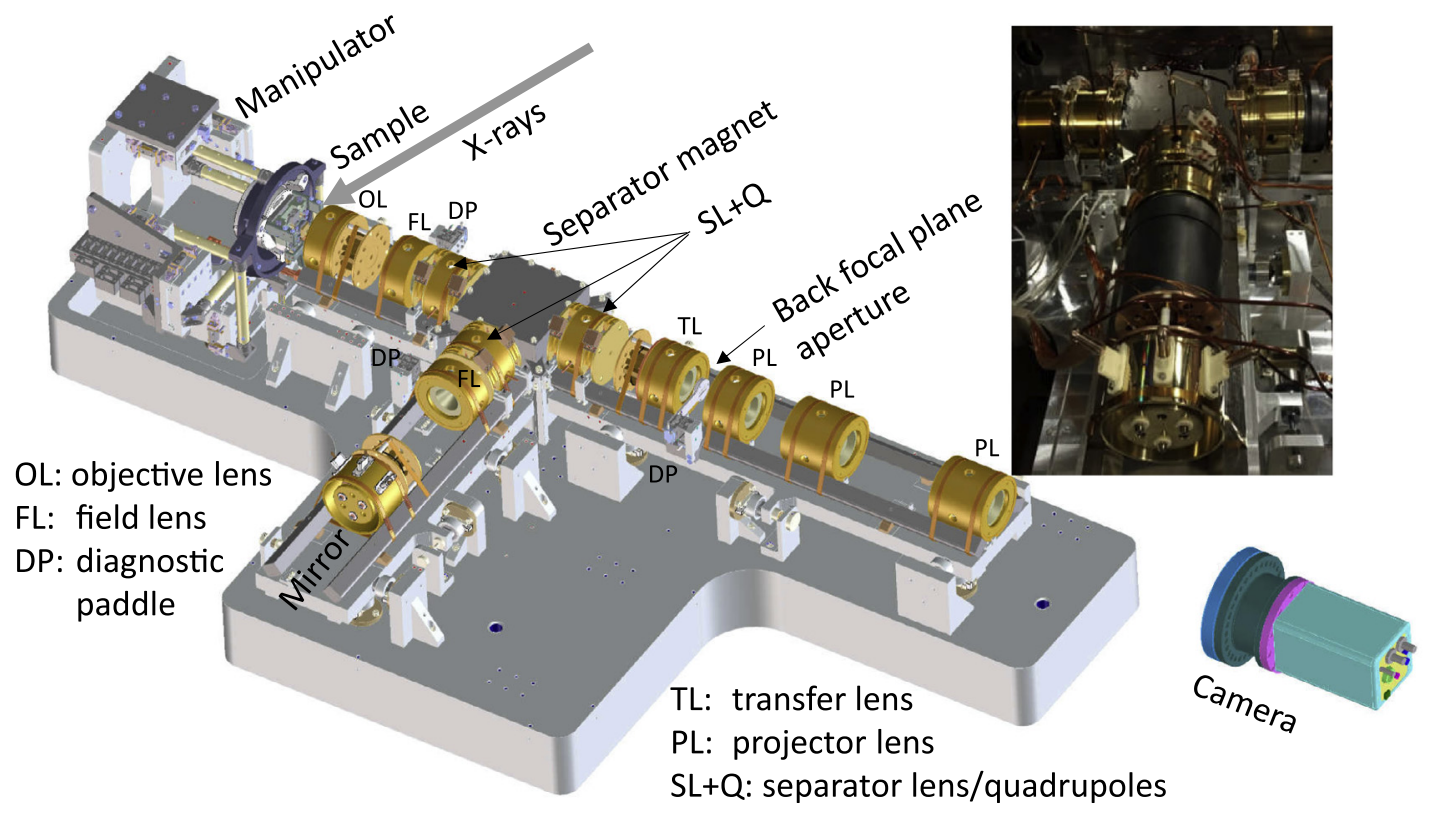

Fig. 1. Layout of the PEEM-3 microscope. The photo shows a view from the mirror towards the separator magnet assembly.

\section{Experimental setup}

Aberration correction of cathode lens electron microscopes is based on concepts developed by Haider et al. [13] and Rempfer et al. [14]. The ALS PEEM-3 microscope uses a tetrode mirror to correct 3rd order spherical and 1st order 1st degree (axial) chromatic aberrations [15,16], Fig. 1. Electron optics, sample manipulator and $90^{\circ}$ beam separator magnet rest on an in-vacuum optical table. A kinematically mounted rail system precisely positions the optical elements. The 5-axis sample manipulator ( $\mathrm{x}, \mathrm{y}, \mathrm{z}$ and two tilt axes) uses in-vacuum, piezo-driven linear stages and a flexure mechanism. Synchrotron x-rays illuminate the sample at an angle of $30^{\circ}$ to the sample surface. Samples can be cooled down to $30 \mathrm{~K}$ using liquid He cooling and heated up to $900 \mathrm{~K}$ using resistive or e-beam heating [17]. Current pulses, voltage pulses, in-situ electromagnetic fields, and in-situ rotation are deployed via special sample holders. All optical elements except for the separator magnet are electrostatic. This minimizes magnetic fields near the sample and prevents heating of the internal support structure. The optical table is fully enclosed inside a mu-metal box. A $1 \mathrm{k} \mathrm{x} 1 \mathrm{k}$ pixel charged coupled device (CCD), fiber-coupled to an electron sensitive phosphor, is used for electron detection.

While using PEEM-3 in straight-through mode or ST-mode, the beam separator magnet is first demagnetized by applying an alternating field of decreasing amplitude. The magnified image of the sample formed by the objective lens is relayed by two round lenses across the demagnetized magnet and further relayed by a transfer lens. An aperture strip with several selectable back focal plane apertures (BFPAs) is located behind the transfer lens. The BFPA blocks off-axis electrons and improves the spatial resolution at the cost of microscope transmission. A projector optics group consisting of three lenses magnifies the image onto the CCD. A 12pole electrostatic deflector assembly directly following the objective lens corrects stigmatic errors, and a set of quadrupoles at the exit of the beam separator steers the beam through the center of the transfer lens.

In aberration-corrected mode or AC-mode, the separator magnet is turned on and bends the electron beam twice, first by $90^{\circ}$ towards the electron mirror and then again by $90^{\circ}$ towards the CCD. The separator magnet assembly consists of a dipole magnet and three groups of optics consisting of a round lens plus two quadrupoles, which together provide astigmatic focusing at $1: 1$ magnification in each quadrant. This simple and tunable design is described in [18]. Diagnostic paddles with slits, pin holes and a TEM grid are located at image planes at the objective-side entrance and the mirror-side exit of the separator assembly. Each quadrant of the separator is chromatic but dispersion is cancelled after the 2nd pass through the magnet following inversion of the image by the mirror. Field lenses near image planes on the objective and mirror side of the separator magnet place a back focal plane in the mirror and remove field aberrations. Stigmatic errors of the objective lens are corrected by a 12-pole deflector near the objective lens. Likewise stigmatic errors of the mirror are corrected by a similar 12-pole deflector near the mirror. The mirror-side 12 pole deflector and a set of quadrupoles near the separator magnet are also used to correctly steer the beam towards the center of the mirror and along the center axis of the separator magnet. Transfer lens, back focal plane apertures and projector lenses are used for resolution improvement and magnification.

The electron mirror was designed to guarantee micron-scale accuracy in the mechanical registration of the electrodes. Phosphor bronze blanks were first mechanically cut and critical surfaces were then diamond-turned to high precision and an optical finish. Concentric registration and electrical isolation of the electrodes was achieved using a chain of sapphire balls placed in concentric grooves, distributing the mechanical forces within the springloaded assembly. A cylindrical metal can shields the internal electrodes and supports the mirror on the in-vacuum rail system. The PEEM-3 mirror with three adjustable electrode potentials is capable of canceling lowest order spherical and chromatic aberrations at a constant imaging distance for a wide range of operating conditions of the microscope. Aberration correction coefficients of the mirror were computed as function of electrode voltage at constant focal length of the mirror and the generated set of correction voltages was then fitted using 5th order polynomial surfaces as function of two free parameters: CC (chromatic correction parameter), 


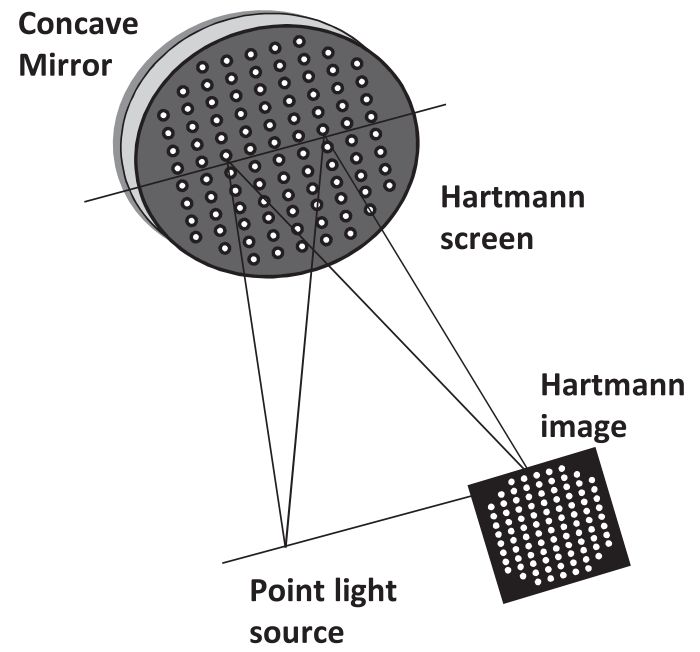

Fig. 2. Classical Hartmann arrangement for the evaluation of large light optics [19].

CS (spherical correction parameter) [16]. The CC and CS correction values can thus be easily adjusted to tune the aberration correction. A small offset voltage applied to the last mirror electrode is being used to tweak the focal length of the mirror.

\subsection{Hartmann measurement of aberration coefficients}

Geometric aberrations, in particular spherical aberrations, limit the performance of X-PEEM. Electrons created by the absorption of high-energy $\mathrm{x}$-rays start from the sample with non-zero energy and a wide angular distribution. First order aberrations, defocus and astigmatism, can be easily countered by adjusting lens voltages and using multipole deflectors. Second order aberrations vanish in a cylinder-symmetrical system and, if present, need to be removed by proper alignment of the electron beam in the microscope. Third order spherical aberrations and axial chromatic aberrations cannot be removed by round electron optics and need to be compensated by the electron mirror.

Approximate aberration coefficients of the frontend optics have been computed for PEEM-3 [16], but in practice we need to experimentally determine the frontend aberrations and find optimal mirror parameters. To this purpose we employ a method derived from the well-known Hartmann technique, which was developed historically for the optical metrology of large light optics, Fig. 2. Light from a point source passes through a hole screen, the Hartmann screen, and is then reflected by the test mirror and finally imaged by a detector near an image plane. Optics imperfections of the mirror warp the displayed dot pattern and the displacements versus the hole coordinate are then fitted by a series of weighted polynomials. A contemporary review of the Hartmann technique can be found in [19]. This technique is similar to the microspot LEED technique discussed in [20] but can also be used in PEEM mode. The Zernike polynomials $Z_{n}^{m}(r, \theta)$ are a commonly used basis for the fitting of optical aberrations and the Zernike coefficients provide quantitative information about the low order geometric aberrations of the tested optics [21,22]. In case of PEEM-3, a 10- $\mu \mathrm{m}$-size back focal plane aperture was scanned across the electron beam, allowing a narrow ray of electrons to pass to the detector. For each position of the BFPA we acquired an image of a test pattern and determined the pattern's location on the detector. Since we measured the image displacements $\mathrm{x}$ and $\mathrm{y}$ as function of aperture position $\mathrm{X}$ and $\mathrm{Y}$ and not the curvature of the wave front we used the derivatives $D_{i}^{X}$ and $D_{i}^{Y}$ of the Zernike polynomials versus $\mathrm{X}$ and
$\mathrm{Y}$ as fit functions:

$$
\begin{aligned}
& x(X, Y)=\sum Z_{i} D_{i}^{X}(X, Y) \\
& D_{3}^{X}=4 X, D_{4}^{X}=2 Y, D_{5}^{X}=-2 X, \\
& D_{6}^{X}=9 X^{2}+3 Y^{2}-2, D_{7}^{X}=6 X Y, \\
& D_{8}^{X}=12 X\left(2 X^{2}+2 Y^{2}-1\right)
\end{aligned}
$$

A corresponding set of fit function was used to fit $y(X, Y)$. The Zernike coefficients $Z_{3}, Z_{4}, Z_{5}$ are the 1 st order defocus and the " $x$ " -oriented and " + " -oriented astigmatisms, $Z_{6}$ and $Z_{7}$ are the 2 nd order coma in $\mathrm{X}$ and $\mathrm{Y}$ direction and $\mathrm{Z}_{8}$ is the 3rd order spherical aberration. The scan pattern of the back focal plane aperture is shown in Fig. 3a. Oversampling improved accuracy, and we measured the image displacement for 28 positions of the BFPA at three different distances from the optical axis to determine six Zernike coefficients, relying on a least square optimization method. We experimented with including higher order aberrations, in particular $Z_{9}, Z_{10}$ (trefoil) and additional third order aberrations but did not observe a reduction in the residue and therefore an improved quality of the fit. Preceding and following the measurement along each circular track we recorded an image at zero BFPA displacement in order to subtract slow sample drift. Typical exposure times were two to ten seconds per image or a few minutes for a full Hartmann measurement. Fig. 3(b) shows images of the test pattern for three $20 \mu \mathrm{m}$ off-axis locations of the BFPA and for the BFPA positioned on the optical axis. Three images are relatively poorly resolved because of the large uncorrected aberrations for off-axis rays. Note that the displayed images were acquired in ST-mode with the aberration corrector turned off. To quantify the displacements we cross-correlated all images with the reference image at zero displacement and determined the shift of the pattern with a nominal accuracy of $1 / 4$ of a pixel (1/4 of a pixel corresponds to $\sim 2.5 \mathrm{~nm}$ ). The same methods were used in ST-mode and in ACmode but the scan pattern was scaled in size to account for the larger magnification of the optics at the BFPA position in AC-mode (Fig. S1). Fig. 3(c) shows the measured and fitted contribution of the spherical aberration to the total image displacement for several CS correction values. These graphs are the result of the Hartmann fit and illustrate the magnitude of the Zernike coefficient $Z_{8}$. A minimal displacement for large BFPA offsets indicates a good correction of the spherical aberration near $\mathrm{CS}=-14,500 \mathrm{~m}$. CS is the dialed-in spherical aberration correction value of the mirror.

Fig. 4 compares the measured and fitted Hartmann displacements, which quantify the geometric image errors, broken up by order for ST-mode (a) and AC-mode (b). In ST-mode 3rd order aberrations dominate (displacements shown in the bottom right panel of Fig. 4(a). Apparently, a non-zero defocus of opposite sign (top right panel) was chosen during microscope alignment so that it partially compensated the image blur due to spherical aberrations (compare the direction of the missing pie slice for 1 st and 3rd order aberrations). 3rd order aberrations are much smaller in AC-mode and an optimal CS correction value of $-14,500 \mathrm{~m}$ was found (bottom right panel of Fig. 4(b). The overall magnitude of the geometrical aberration caused displacements is also much smaller (black line, top left panel of $4 \mathrm{~b}$ in comparison to $4(\mathrm{a})$. 2nd order aberrations are small in ST and in AC mode, as expected for a well aligned optics. The size of the residue of the fit (red dots) indicates that the remaining aberrations are smaller or of similar magnitude as the measurement error of the Hartmann method.

Repeating the optics alignment process following different recipes showed that 2 nd order aberrations (coma) can become dominant in AC-mode, for example when the electron beam does not enter the mirror on axis. Proper alignment of the beam in lenses and especially in the mirror are therefore crucial to achieve a high performance of the aberration corrector. The described 
a)

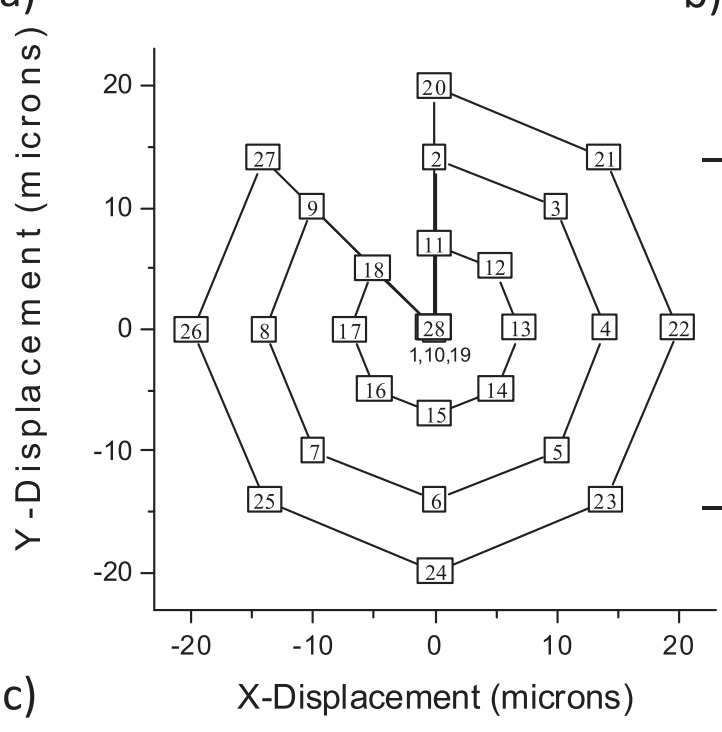

b)

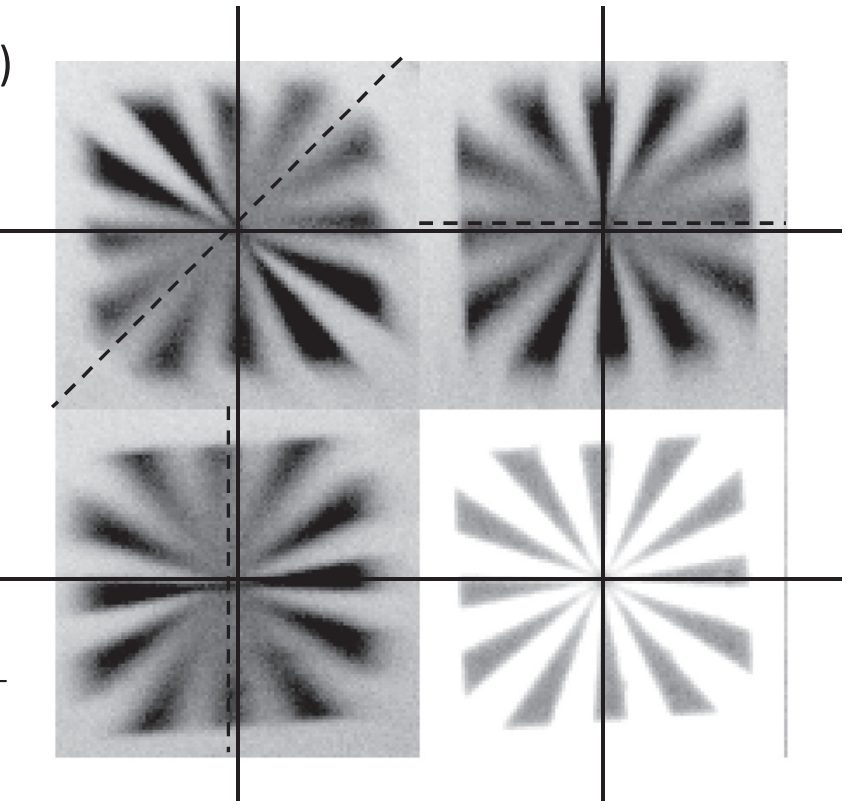

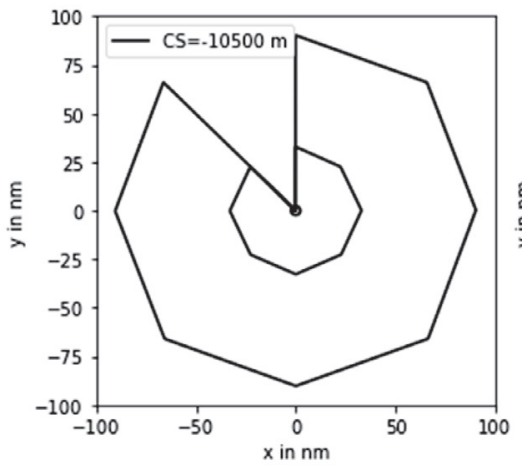
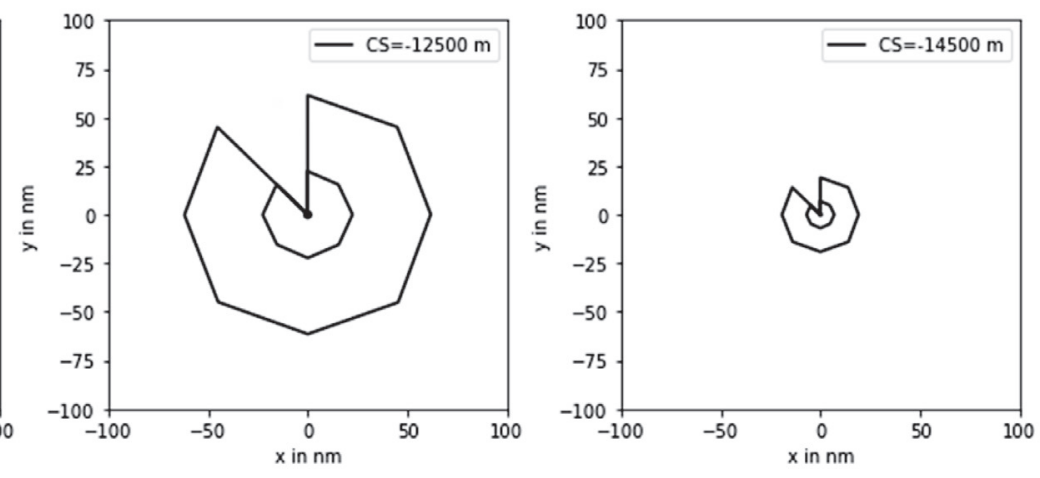

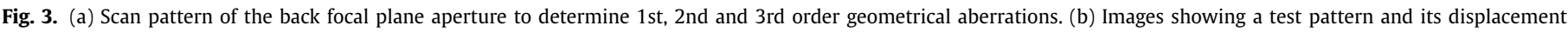

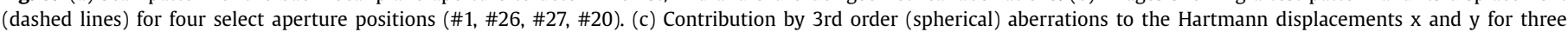
different CS correction values in AC-mode.

Table 1

1st to 3rd order aberration coefficients and contribution of the spherical aberration to the beam spread on the detector.

\begin{tabular}{lllll}
\hline & $\begin{array}{l}\text { 1st order } \\
(\mathrm{nm} / \mu \mathrm{m})\end{array}$ & $\begin{array}{l}\text { 2nd order } \\
\left(\mathrm{nm} / \mu \mathrm{m}^{2}\right)\end{array}$ & $\begin{array}{l}\text { 3rd order } \\
\left(\mathrm{nm} / \mu \mathrm{m}^{3}\right)\end{array}$ & $\begin{array}{l}\text { beam spread } \\
(\mathrm{nm})\end{array}$ \\
\hline $\begin{array}{l}\text { ST-mode } \\
\text { AC-mode }\end{array}$ & 0.58 & 0.013 & 0.00038 & 777 \\
& 0.38 & 0.008 & 0.00004 & 82 \\
\hline
\end{tabular}

Hartmann method is able to differentiate between errors caused by an incorrect objective lens voltage (defocus), poor alignment (astigmatism and coma), and uncompensated spherical aberrations and provides an accurate method to qualify the optics of the aberration corrected X-PEEM microscope.

Table 1 lists the aggregated aberration coefficients by order for ST-mode and AC-mode. Same order aberrations (1st order: Z3-Z5, 2nd order: Z6-Z7, 3rd order: Z8) were added in quadrature. To take into account the 1.63 times larger magnification of AC-mode at the BFPA position (Fig. S1), the aberration coefficients for ST-mode were scaled by $1 / 1.63$ in 1 st order, $1 / 1.63^{2}$ in 2 nd order and by $1 / 1.63^{3}$ in 3rd order. This allows us to compare ST-mode and ACmode directly. We list the aberration coefficients in units of $\mathrm{nm}$ (image displacement) per $\mu \mathrm{m}^{\mathrm{n}}$ (aperture displacement, $\mathrm{n}=1,2,3$ is the aberration order). We list the aberration coefficients as function of the beam angle at the transfer lens in Table 2 .
Table 2

1 st to 3rd order aberration coefficients and contribution of the spherical aberration to the beam spread on the detector as function of the beam angle at the transfer lens.

\begin{tabular}{llll}
\hline & $\begin{array}{l}\text { 1st order in } \\
\mu \mathrm{m} / \mathrm{rad}\end{array}$ & $\begin{array}{l}\text { 2nd order in } \\
\mathrm{mm} / \mathrm{rad}^{2}\end{array}$ & $\begin{array}{l}\text { 3rd order in } \\
\mathrm{m} / \mathrm{rad}^{3}\end{array}$ \\
\hline $\begin{array}{l}\text { ST-mode } \\
\text { AC-mode }\end{array}$ & 19 & 14 & 14 \\
& 12 & 9 & 1.5 \\
\hline
\end{tabular}

While the compounded 1 st and 2 nd order errors are of similar magnitude in ST- and AC-mode, we measure an order of magnitude reduced 3rd order coefficient, demonstrating the ability of the mirror corrector to cancel and suppress the effect of spherical aberrations. It is noteworthy that the mirror corrector does not increase other low order aberrations-they are in fact about $40 \%$ smaller in this experiment-which demonstrates the high quality of the mechanical and optical alignment of the mirror arm optics. To put those values into context we compute and list the approximate displacement of electron rays passing the BFPA plane at a distance from the optical axis of $44 \mu \mathrm{m}$ in AC-mode and $27 \mu \mathrm{m}$ in ST-mode in the column labeled "beam spread". These numbers represent the median beam radius: half of the electrons pass within a disc of this size, Fig. S1. The displacements are presented in object coordinates for a virtual magnification of the microscope of 1 . They 

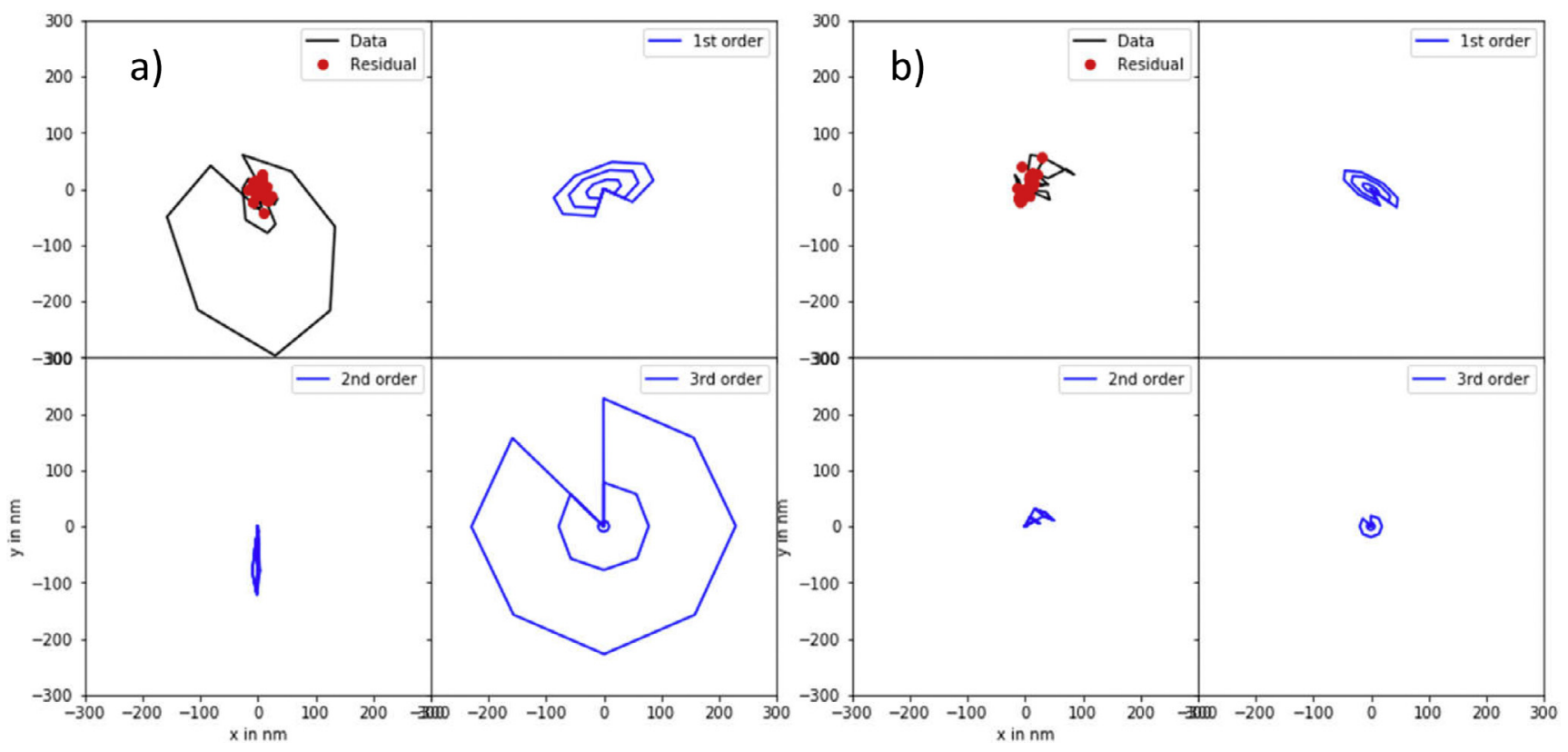

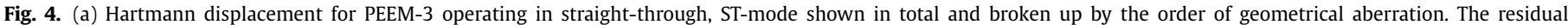

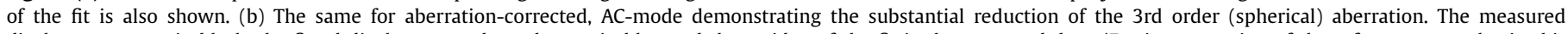

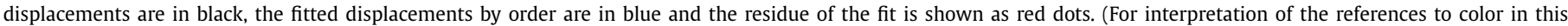
figure legend, the reader is referred to the web version of this article.)

can be interpreted as the median radius of the aberrated image of a point source. As expected, spherical aberrations cause very large image errors of hundreds of nanometers in ST-mode. The beam spread at the detector extends over more than $700 \mathrm{~nm}$, with $50 \%$ of the electrons landing outside a disc of this radius. Aberration correction reduces the spread at the detector due to spherical aberrations to less than $100 \mathrm{~nm}$. This should allow us to achieve $100 \mathrm{~nm}$ level spatial resolution, even without the use of a back focal plane aperture and at $100 \%$ transmission of the electron optics, as predicted in [15]. It must be noted that we have not considered the effect of axial chromatic aberrations here, which can be large for an X-ray PEEM. The PEEM-3 electron mirror is expected to counter these as well, as images of test patterns will show.

\subsection{Demonstration of aberration correction on test patterns}

Fig. 5 shows X-PEEM images of an elbow-shaped line pattern, which were acquired using element contrast at the Ni edge without using a contrast-enhancing BFPA. The patterns were manufactured by e-beam lithography in Ni on a Si substrate [17]. The width of the lines was $150 \mathrm{~nm}$ and areas covered by Ni appeared bright. Images were exposed for $10 \mathrm{~s}$ and a sample voltage of $15 \mathrm{kV}$ was used, which is a conservative value. For very high resolution experiments we typically operate PEEM-3 at up to $20 \mathrm{kV}$ or even at higher sample voltages, resulting in an approximately proportional improvement in resolution and at the same time improved detector signal. The image brightness was similar in ST- and ACmode, indicating that the microscope transmission in ST-mode and AC-mode were the same. However, we chose to insert an image plane aperture of $100 \mu \mathrm{m}$ diameter in the objective lens column when using AC-mode in order to reduce the electron density at the electron turnaround point in the mirror and prevent space charge blurring, which was severe otherwise. This image plane aperture restricted the field of view to about $8 \mu \mathrm{m}$ but did not lower the transmission of the microscope within the field of view. We observed that the image plane aperture upstream of separator magnet and mirror effectively eliminated space charge effects for low to moderate $\mathrm{x}$-ray flux (see also Fig. S2). Intensity profiles across
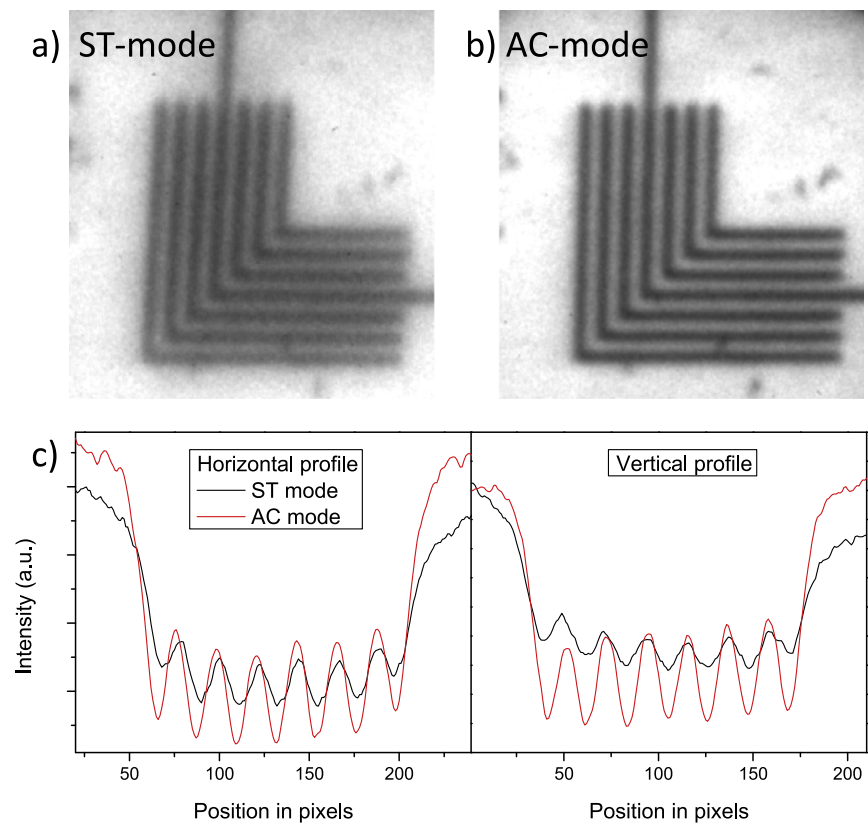

Fig. 5. $150 \mathrm{~nm}$ line width, elbow-shaped lithographic patterns imaged using (a) STmode" and b) AC-mode at 100\% transmission (back focal plane aperture removed). (c) Line profiles across the vertical and horizontal lines.

the pattern along the horizontal and the vertical direction show a 2-3 times reduced contrast without using aberration correction and a wide halo, while the AC-mode images look crisp and the $\mathrm{Ni}$ lines appear at higher contrast. The line intensity within the pattern is still below the intensity of areas far from the pattern, which means that electrons with large transverse momentum (high initial energy and large emission angle) are still not optimally focused, even using AC-mode. This is likely the result of higher order aberrations that are not corrected and possibly of not optimally chosen CC and CS correction values. In particular, choosing an optimal CC 
a)

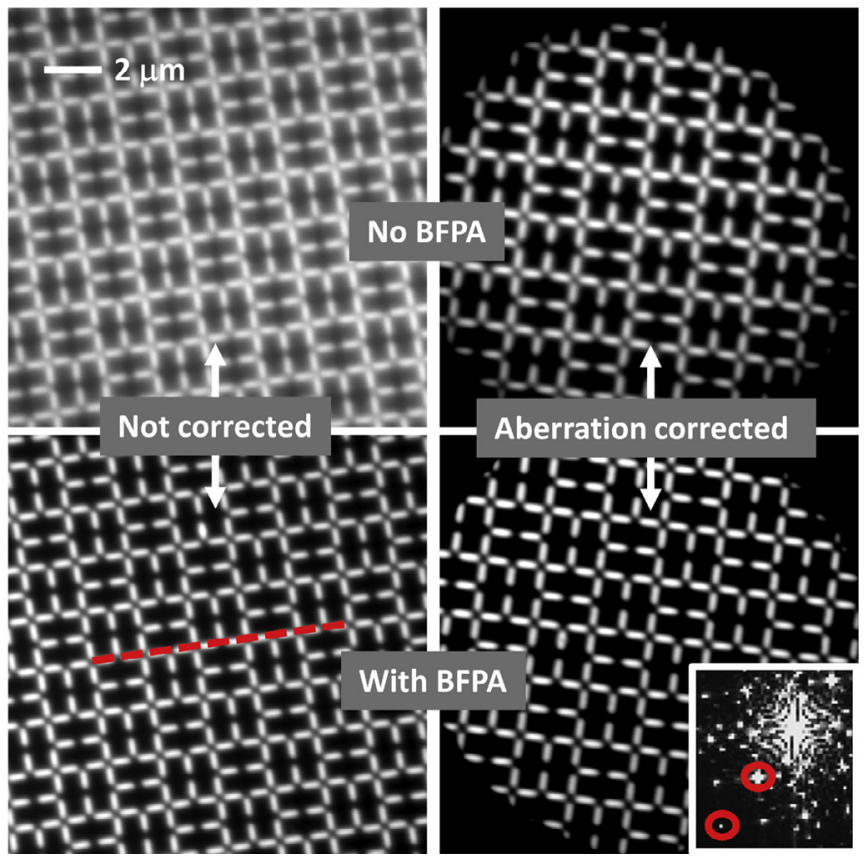

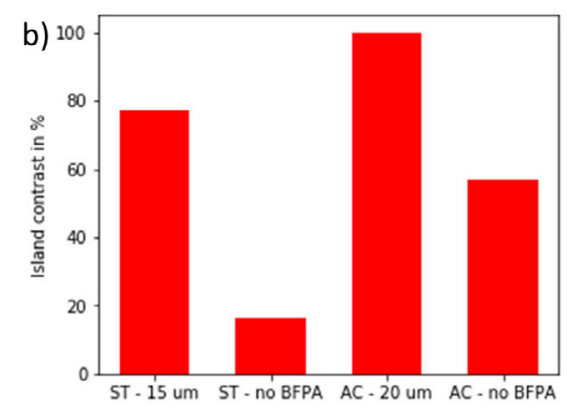

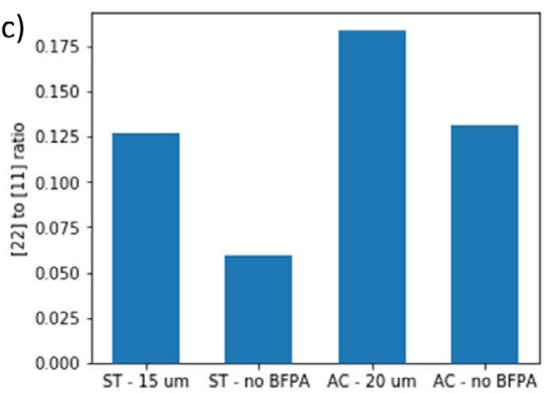

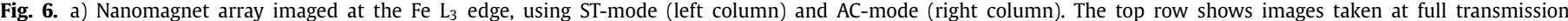

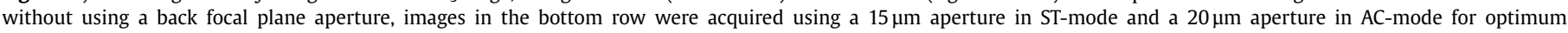

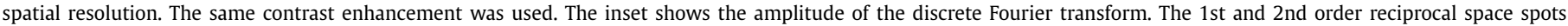

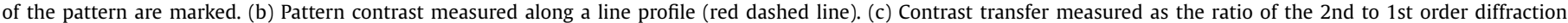
spot. (For interpretation of the references to color in this figure legend, the reader is referred to the web version of this article.)

value is not straightforward when the source is polychromatic and no energy filter is used. A range of CC values was tried and the images with best resolved features are shown here.

In order to quantify the relative contrast transfer of ST-mode and AC-mode we imaged a nano-magnet lattice of $2.8 \mathrm{~nm}$ thick Ni80Fe20 rectangular islands at the $\mathrm{Fe}_{\mathrm{L}}$ edge, Fig. 6 [23]. The islands are $470 \times 170 \mathrm{~nm}$ in size on a lattice with $600 \mathrm{~nm}$ spacing. The islands appear bright, while the Si substrate appears dark. Uncorrected images (left column) are compared with aberrationcorrected images (right column), while not using a BFPA (top row) and while using a contrast enhancing BFPA (bottom row). The $100 \mu \mathrm{m}$ size image plane aperture upstream of the mirror is visible in the AC-mode images and was again used to prevent space charge blurring. For the high resolution images, small back focal plane apertures of $15 \mu \mathrm{m}$ (ST-mode) and $20 \mu \mathrm{m}$ (AC-mode) were chosen. The top row images illustrates the primary function of the mirror corrector in an X-PEEM at high transmission: The mirror removes the large diffuse background of badly aberrated, off-axis rays and dramatically increases the image contrast. The relative image contrast was determined using two methods, the first relied on measuring the variation of the image intensity along a line profile (dashed red line), the second was based on measuring the relative strength of the 2nd and 1st order reciprocal space spots of the periodic lattice in Fourier space. The amplitude of higher frequency components in Fourier space decreases with decreasing contrast transfer, therefore the intensity ratio of different order spots can be used to compare the optical quality of images of such a lattice structure. The inset in Fig. 6a shows the Fourier transform of a real space X-PEEM image with the 1 st and 2 nd order spots marked by red circles. The position of the 2nd order spot in Fourier space corresponds to a real space distance or line width of $106 \mathrm{~nm}$.

The bar diagram in Fig. 6(b) lists the pattern to gap contrast along a row of nanomagnets for four tested X-PEEM modes. The data were normalized to the value measured using a $20 \mu \mathrm{m}$ BFPA in AC-mode, which showed the highest contrast. In ST mode and using a BFPA the contrast is reduced to about $75 \%$, it is about $55 \%$ at optimal microscope transmission when using AC-mode and no $\mathrm{BFPA}$, and it is very low, around $15 \%$, when using neither aberration correction nor a BFPA. We observe that aberration correction improves the image quality and contrast transfer considerably when no BFPA is used (15\% to 55\% relative contrast). This improvement is less striking but still visible ( $75 \%$ to $100 \%$ relative contrast) when inserting a BFPA, which by itself reduces the contribution of badly aberrated rays. A similar trend is visible in Fig. 6(c) which summarizes the Fourier space analysis. Fig. 6(c) shows a sizeable increase in the relative intensity of the 2 nd order reciprocal space spot when the AC correction is turned on, with and without a BFPA. We have repeated this analysis using the 3rd order reciprocal space spot and obtained similar results. According to these measurements AC-mode without a BFPA is even competitive with ST-mode using a small BFPA. In summary, both analysis methods reveal a large improvement in image contrast at high microscope transmission and a smaller but still measureable improvement at low transmission, when a BFPA is used.

We also studied the performance of the aberration correction in magnetic microscopy experiments. Fig. 7 shows $\mathrm{X}$-ray magnetic circular dichroism (XMCD) images of an array of $100 \mathrm{~nm}$ diameter magnetic islands on a $150 \mathrm{~nm}$ period honey comb lattice [24]. The distance between the $10 \mathrm{~nm}$ thick $\mathrm{Ni}_{80} \mathrm{Fe}_{20}$ discs is $50 \mathrm{~nm}$. Magnetic imaging of such disc patterns is challenging because the magnetic contrast is around 10 times weaker than the element contrast employed in the previous tests and the distance between the islands is small and approaches the resolution limit of PEEM-3. Ten images were acquired at the $\mathrm{Fe}_{\mathrm{L}} \mathrm{L}_{3}$ edge at $707 \mathrm{eV}$ for each circular $\mathrm{X}$-ray polarization, averaged, and then divided. The resulting XMCD images are shown at the same level of contrast using ST-mode on the left, using AC-mode on the right, without using a BFPA in the top row and using BFPAs to improve contrast at the cost of transmission in the bottom row. The transmission without using a BFPA is nominally $100 \%$, while it is reduced to $14 \%$ using a $20 \mu \mathrm{m}$ diameter aperture in ST-mode and to $28 \%$ using a larger, $50 \mu \mathrm{m}$ aperture in AC-mode. The magnetic patterns are well resolved in both AC- 


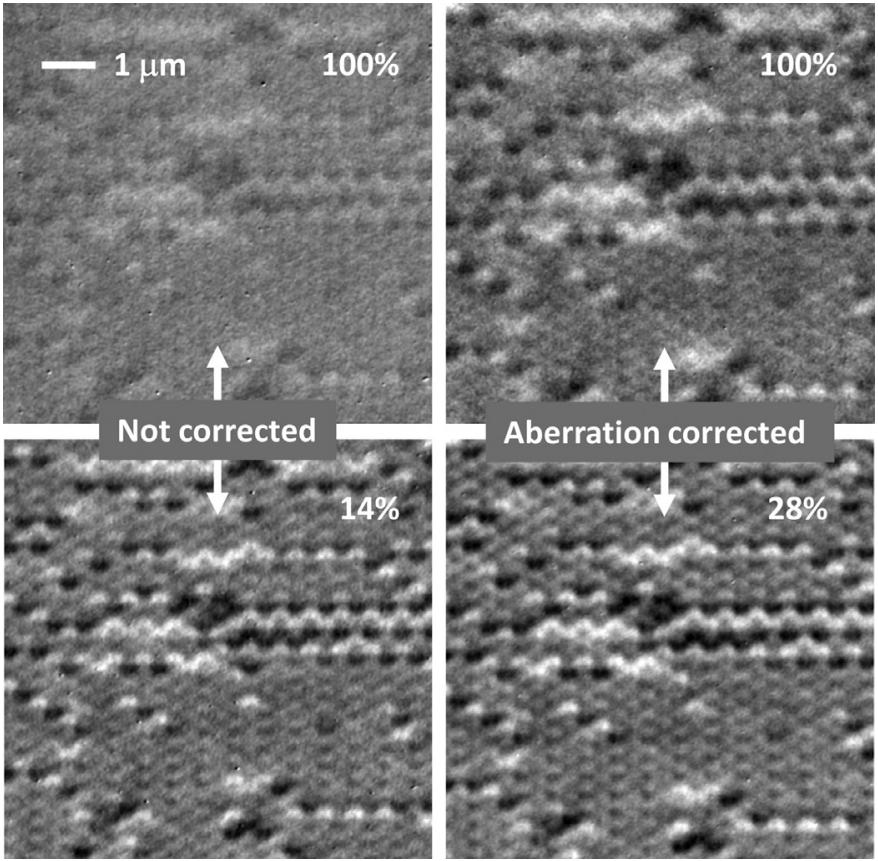

Fig. 7. XMCD images of a honey comb pattern of $100 \mathrm{~nm}$ sized circular magnetic islands imaged using (left) ST-mode and (right) AC-mode. The percentages quantify the transmission of the microscope for the selected back focal plane apertures. The same contrast enhancement was used when displaying the images.

mode and ST-mode when using a BFPA but a twice larger transmission can be chosen when the aberration correction is employed. Even without a contrast enhancing BFPA in use, aberration corrected images have sufficient quality for analysis while the uncorrected images are of poor quality and have very low contrast. The total exposure time needed to measure the aberration corrected domain image in high transmission mode (no BFPA) was $10 \mathrm{~s}$ per polarization ( $1 \mathrm{~s}$ per exposure) at a field of view of $8 \mu \mathrm{m}$ and at about $30 \%$ of the maximal available x-ray flux. The images demonstrate that aberration correction enables imaging of features with sub $100 \mathrm{~nm}$ scale separation at higher transmission of the electron optics. Short exposure times become possible even when operating at very high magnification.

\section{Conclusions}

We have shown that aberration correction significantly improves the resolution of the PEEM-3 electron optics, in particular, when a high microscope transmission and efficiency are needed, e.g., for fast (dynamic) imaging or to prevent x-ray damage. Hartmann measurements show that spherical aberrations, which are massive for off-axis electrons in an uncorrected PEEM, are reduced by an order of magnitude. Demonstration measurements on samples that cover important use cases of the instrument show that chromatic and spherical aberrations are well cancelled by the electron mirror and allow us to image $100 \mathrm{~nm}$ scale and smaller features with good contrast and low background even at high transmission. The mirror does not increase other low order geometrical aberrations, proving the good alignment of the optics. We have shown that Hartmann measurements are critical for tuning the microscope and for qualifying the optics of an X-PEEM.

\section{Acknowledgments}

This research used resources of the Advanced Light Source, which is a DOE Office of Science User Facility under contract no.
DE-AC02-05CH11231. R.S. and N.K. acknowledge support from the U.S. Department of Energy, Office of Science, Basic Energy Sciences, Materials Sciences and Engineering Division under Contract No. DE-AC02-05-CH11231 (NEMM program MSMAG). The magnetic lattice samples were prepared by J.D. Watts, A.M. Albrecht and C. Leighton at the University of Minnesota and Y. Lao and J. Sklenar at the University of Illinois. We would like to thank D. Deboer and B. Conners for the fabrication and mechanical alignment of the electron optics of the mirror corrector. The elbow test pattern was produced by W. Chao and E. Anderson at the LBNL Center for X-ray Optics.

\section{Supplementary materials}

Supplementary material associated with this article can be found, in the online version, at doi:10.1016/j.ultramic.2018.03.010.

\section{References}

[1] A. Scholl, J. Stöhr, J. Lüning, J.W. Seo, J. Fompeyrine, H. Siegwart, J.P. Locquet, F. Nolting, S. Anders, E.E. Fullerton, M.R. Scheinfein, H.A. Padmore, Observation of antiferromagnetic domains in epitaxial thin films, Science 287 (2000) 1014-1016.

[2] S.B. Choe, Y. Acremann, A. Scholl, A. Bauer, A. Doran, J. Stohr, H.A. Padmore, Vortex core-driven magnetization dynamics, Science 304 (2004) 420-422.

[3] T. Schmidt, S. Heun, J. Slezak, J. Diaz, K.C. Prince, G. Lilienkamp, E. Bauer, SPELEEM: combining LEEM and spectroscopic imaging, Surf. Rev. Lett. 5 (1998) 1287-1296.

[4] J. Stöhr, Y. Wu, B.D. Hermsmeier, M.G. Samant, G.R. Harp, S. Koranda, D. Dunham, B.P. Tonner, Element-specific magnetic microscopy with circularly polarized x-rays, Science 259 (1993) 658-661.

[5] S. Anders, H.A. Padmore, R.M. Duarte, T. Renner, T. Stammler, A. Scholl, M.R. Scheinfein, J. Stöhr, L. Seve, B. Sinkovic, Photoemission electron microscope for the study of magnetic materials, Rev. Sci. Instrum. 70 (1999) 3973-3981.

[6] G. De Stasio, L. Perfetti, B. Gilbert, O. Fauchoux, M. Capozi, P. Perfetti, G. Margaritondo, B.P. Tonner, MEPHISTO spectromicroscope reaches $20 \mathrm{~nm}$ lateral resolution, Rev. Sci. Instrum. 70 (1999) 1740-1742.

[7] G. Schönhense, H. Spiecker, Correction of chromatic and spherical aberration in electron microscopy utilizing the time structure of pulsed excitation sources, J. Vac. Sci. Technol. B 20 (2002) 2526-2534.

[8] H. Ishiwata, Y. Acremann, A. Scholl, E. Rotenberg, O. Hellwig, E. Dobisz, A. Doran, B. Tkachenko, A. Fokin, P. Schreiner, J. Dahl, R. Carlson, N. Melosh, Z. Shen, H. Ohldag, Diamondoid coating enables disruptive approach for chemical and magnetic imaging with $10 \mathrm{~nm}$ spatial resolution, Appl. Phys. Lett. 101 (2012).

[9] T. Schmidt, U. Groh, R. Fink, E. Umbach, XPEEM with energy-filtering: advantages and first results from the smart project, Surf. Rev. Lett. 9 (2002) 223-232.

[10] R.M. Tromp, J.B. Hannon, W. Wan, A. Berghaus, O. Schaff, A new aberrationcorrected, energy-filtered LEEM/PEEM instrument II. Operation and results, Ultramicroscopy 127 (2013) 25-39.

[11] R. Konenkamp, R.C. Word, G.F. Rempfer, T. Dixon, L. Almaraz, T. Jones, 5.4 nm spatial resolution in biological photoemission electron microscopy, Ultramicroscopy 110 (2010) 899-902.

[12] T. Schmidt, A. Sala, H. Marchetto, E. Umbach, H.J. Freund, First experimental proof for aberration correction in XPEEM: resolution, transmission enhancement, and limitation by space charge effects, Ultramicroscopy 126 (2013) 23-32.

[13] M. Haider, H. Rose, S. Uhlemann, B. Kabius, K. Urban, Towards $0.1 \mathrm{~nm}$ resolution with the first spherically corrected transmission electron microscope, J. Electron. Microsc. 47 (1998) 395-405.

[14] G.F. Rempfer, D.M. Desloge, W.P. Skoczylas, O.H. Griffith, Simultaneous correction of spherical and chromatic aberrations with an electron mirror: an electron optical achromat, Microsc.Microanal. 3 (1997) 14-27.

[15] J. Feng, E. Forest, A.A. MacDowell, M. Marcus, H. Padmore, S. Raoux, D. Robin, A. Scholl, R. Schlueter, P. Schmid, J. Stohr, W. Wan, D.H. Wei, Y. Wu, An X-ray photoemission electron microscope using an electron mirror aberration corrector for the study of complex materials, J. Phys. Condens.Matter 17 (2005) S1339-S1350.

[16] W. Wan, J. Feng, H.A. Padmore, D.S. Robin, Simulation of a mirror corrector for PEEM3, Nucl. Instrum. Methods Phys. Res. Sect. A 519 (2004) 222.

[17] A. Doran, M. Church, T. Miller, G. Morrison, A.T. Young, A. Scholl, Cryogenic PEEM at the advanced light source, J. Electr. Spectrosc. Relat. Phenom. 185 (2012) 340-346.

[18] W. Wan, J. Feng, H.A. Padmore, A new separator design for aberration corrected photoemission electron microscopes, Nucl. Instrum. Methods Phys. Res. Sect. A-Accel. Spectrom. Detect. Assoc. Equip. 564 (2006) 537-543.

[19] D. Malacara-Hernandez, D. Malacara-Doblado, What is a Hartmann test? Appl. Opt. 54 (2015) 2296-2301. 
[20] R.M. Tromp, Characterization of the cathode objective lens by real-space microspot low energy electron diffraction, Ultramicroscopy 130 (2013) 2-6.

[21] V. Lakshminarayanan, A. Fleck, Zernike polynomials: a guide, J. Mod. Opt. 58 (2011) 545-561.

[22] F. Zernike, Diffraction theory of the knife-edge test and its improved form, the phase-contrast method, Mon. Not. R. Astron. Soc. 94 (1933) 0377-0384.
[23] I. Gilbert, Y.Y. Lao, I. Carrasquillo, L. O’Brien, J.D. Watts, M. Manno, C. Leighton, A. Scholl, C. Nisoli, P. Schiffer, Emergent reduced dimensionality by vertex frustration in artificial spin ice, Nature Phys. 12 (2016) 162-+.

[24] S. Velten, R. Streubel, A. Farhan, N. Kent, M.Y. Im, A. Scholl, S. Dhuey, C. Behncke, G. Meier, P. Fischer, Vortex circulation patterns in planar microdisk arrays, Appl. Phys. Lett. 111 (2017) 059901. 